\title{
Effects of long-term in vitro exposure to phosphodiesterase type-3 inhibitors on follicle and oocyte development
}

\author{
D Nogueira, R Cortvrindt, B Everaerdt and J Smitz \\ Follicle Biology Laboratory, Vrije Universiteit Brussel (VUB), Laarbeeklaan, 101, Brussels, 1090, Belgium \\ Correspondence should be addressed to D Nogueira; Email: daniela_nogueira@yahoo.com
}

\begin{abstract}
Germinal vesicle (GV)-stage oocytes retrieved from antral follicles undergo nuclear maturation in vitro, which typically occurs prior to cytoplasmic maturation. Short-term culture with meiotic inhibitors has been applied to arrest oocytes at the GV stage aiming to synchronize nuclear and ooplasmic maturity. However, the results obtained are still far from the in vivo situation. In order to acquire competence, immature oocytes may require meiotic arrest in vitro for a more extended period. The phosphodiesterase type 3-inhibitor (PDE3-I) is a potent meiotic arrester. The effects of a prolonged culture with PDE3-I on oocyte quality prior to and after reversal from the inhibition are not known. This study tested the impact of long-term in vitro exposure of two PDE3-Is, org9935 and cilostamide, on oocytes using a mouse follicle culture model. The results showed that PDE3-I (maximum of $10 \mu \mathrm{M}$ ) during a 12-day culture of follicle-enclosed oocytes did not alter somatic cell proliferation, differentiation or follicle survival. In addition, the steroid production profile was not significantly modified by a 12-day exposure to PDE3-I. The recombinant human chorionic gonadotrophin/recombinant human epidermal growth factor stimulus induced a characteristic normal progesterone peak of luteinization and normal mucification of the cumulus cells, while the enclosed oocyte remained blocked at the GV stage. In vitro maturation of denuded or cumulus-enclosed oocytes derived from org9935- or cilostamide-exposed follicles progressed through meiosis and formed morphologically normal meiotic spindles with chromosomes properly aligned at the equator. In conclusion, long-term culture with PDE3-I was harmless to somatic cell function, differentiation, oocyte growth and maturation. Our results suggested that PDE3-I can be applied when extended oocyte culture is required to improve ooplasmic maturation.

Reproduction (2005) 130 177-186
\end{abstract}

\section{Introduction}

Production of a developmentally competent oocyte depends on gonadotrophin actions during follicular development, granulosa cell differentiation and oocytegranulosa cell interactions, which are intimately associated and interdependent (Buccione et al. 1990, Allworth \& Albertini 1993, Canipari 2000). During oocyte development within the antral follicle, the oocyte is maintained in a state of nuclear arrest at prophase I. This is established by interaction with granulosa cells, which provide the oocyte with appropriate levels of CAMP, and via meiotic inhibitory substances in follicular fluid (e.g. hypoxanthine). This state of natural arrest brings about optimal synchronization between oocyte nuclear and cytoplasmic maturation. When fully grown germinal vesicle (GV)-stage oocytes are retrieved from antral follicles, they spontaneously resume meiosis in culture. Generally, oocyte nuclear maturation in vitro occurs before ooplasm has reached full maturity and, because of deficiency in ooplasm factors and/or organization, oocytes from small/intermediate antral follicles are developmentally incompetent (Eppig et al. 1994).

Meiotic arresters can be added during GV oocyte culture to promote ooplasmic maturation in vitro by allowing an extended period of culture. With this aim, the use of cell- and target-specific molecules is advantageous. Phosphodiesterase (PDE) subtypes are differentially expressed in the somatic and germ cell compartments of the follicle (Tsafriri et al. 1996, Park et al. 2003). The oocyte-specific PDE that controls cyclic nucleotide accumulation is PDE type 3A (PDE3A) (Tsafriri et al. 1996, Shitsukawa et al. 2001). PDE3A is involved in in vitro induced and spontaneous resumption of meiosis in rat cumulus-enclosed oocytes (CEO): PDE3A activity is increased after stimulation by human chorionic gonadotrophin (hCG) and $2 \mathrm{~h}$ after oocyte isolation. In both situations, this increase in PDE3A activity precedes oocyte nuclear maturation (Richard et al. 2001). Soluble forms of PDE3A exist in oocytes (Shitsukawa et al. 2001), but the mechanisms of activation of the PDE3A remain unknown. Studies aiming 
to develop a new contraceptive strategy have shown that inhibition of PDE3A blocks oocyte meiosis in several species in vivo and in vitro (Tsafriri et al. 1996, Wiersma et al. 1998, Jensen et al. 2002, Mayes \& Sirard 2002, Nogueira et al. 2003a).

Changes in CAMP levels are responsible for temporal regulation of CAMP-dependent protein kinases (PKA). The increase in CAMP levels, in oocytes cultured with CAMPelevating agents and in oocytes from PDE3-deficient mice, potentiates PKA activity, and inhibits meiosis progression by preventing activation of maturation-promoting factor (MPF) and mitogen-activating protein kinase (MAPK) (Dekel et al. 1996, Bilodeau-Goeseels 2003, Masciarelli et al. 2004).

PDE3 inhibitor (PDE3-I) can be applied as a strategy to arrest oocyte meiosis and promote in vitro oocyte cytoplasmic maturation (Nogueira et al. 2003 b, Thomas et al. 2004). To date, PDE3-Is have been applied only during short culture periods (Jensen et al. 2002, Mayes \& Sirard 2003). Use of specific PDE3-I has been shown to enhance oocyte quality, but suboptimally compared with in vivo matured oocytes (Thomas et al. 2002). It seems that when rodent oocytes are meiotically inhibited with the PDE3-I for a time-frame equivalent to the in vivo situation, in vitro oocyte development better approaches the quality of in vivo matured oocytes (Nogueira et al. 2003b). We have therefore speculated that in order to acquire competence, retrieved GV-stage oocytes from large species and humans should be meiotically arrested for a prolonged period of time, comparable with the time taken in vivo to reach the preovulatory stage. The effects of a prolonged exposure (longer than $48 \mathrm{~h}$ ) of immature oocytes with PDE3-I are unknown. As a first step to verify this hypothesis, we intended to test whether the use of PDE3-I for a prolonged period could cause any undesirable effect on oocyte development prior to and after removal from the inhibitor. The mouse follicle culture system (Cortvrindt \& Smitz 2002) approaches physiological conditions, maintains granulosa cell-oocyte interactions and enables exposure of in vitro follicles to PDE3-I for up to 12 days. As the oocyte influences its microenvironment by regulating gene expression in granulosa cells and the organization of the follicle (Elvin et al. 1999, Joyce et al. 2001, Eppig et al. 2002), potentially detrimental effects of inhibition of PDE3 on, for example, steroidogenesis, must be ruled out. Therefore, an investigation was undertaken on the direct or indirect effects of a continuous long-term exposure of follicles to PDE3-I, analysing follicle development, steroidogenesis, oocyte growth and the maturation process.

\section{Materials and Methods}

\section{Animals}

All mice used were F1 hybrids $(\mathrm{C} 57 \mathrm{Bl} / 6 \mathrm{~J} \times \mathrm{CBA} / \mathrm{ca})$, housed and bred according to national legislation for animal care. This study was approved by the Institutional
Ethical Commission for animal experiments (project no. 01-395-1).

\section{Isolation and culture of preantral follicles}

The follicle culture system used was developed by Cortvrindt et al. (1998). Briefly, early preantral follicles with a diameter between $100 \mu \mathrm{m}$ and $130 \mu \mathrm{m}$ were mechanically isolated from ovaries of 14-day-old mice. Follicles were collected in washing medium consisting of L15 Leibovitz-glutamax Life Technologies, Merelbeke, Belgium supplemented with $10 \%$ fetal calf serum (FCS), $100 \mathrm{U} / \mathrm{ml}$ penicillin and $100 \mathrm{mg} / \mathrm{ml}$ streptomycin. The culture medium (control media) used consisted of $\alpha$-minimum essential medium with glutamax (Life Technologies, Merelbeke, Belgium), supplemented with 5\% heat-inactivated FCS, ITS $(5 \mu \mathrm{g} / \mathrm{ml}$ insulin, $5 \mu \mathrm{g} / \mathrm{ml}$ transferrin and $5 \mathrm{ng} / \mathrm{ml}$ sodium selenite) (Sigma, Bornem, Belgium) and $100 \mathrm{mIU} / \mathrm{ml}$ recombinant follicle-stimulating hormone $(\mathrm{rFSH})$ and $10 \mathrm{mIU} / \mathrm{ml}$ recombinant luteinizing hormone (LH) (all recombinant gonadotrophins were a gift from Ares Serono International, Geneva, Switzerland). Follicles were cultured individually in 20 microdroplets of $10 \mu \mathrm{l}$ medium in $60 \mathrm{~mm}$ Petri dishes covered with $5 \mathrm{ml}$ mineral oil (Sigma). On day 2 of the culture, $10 \mu \mathrm{l}$ fresh medium was added and thereafter half of the medium was replaced every other day. Dishes of all culture steps were placed in a humidified atmosphere of $37^{\circ} \mathrm{C}, 5 \% \mathrm{CO}_{2}$ in air.

\section{PDE3-IS}

Two PDE3-Is, org9935 (a gift from Organon, Oss, The Netherlands) and cilostamide (ICN Biomedicals, AsseRelegem, Belgium) were used dissolved in dimethyl sulphoxide (DMSO). The PDE-I was added in $1 \mu \mathrm{M}$ and $10 \mu \mathrm{M}$ final concentrations to the control medium during the follicle culture period. The highest concentration of DMSO used was $0.1 \%$. The eventual influence of DMSO $(0.1 \%)$ applied during the entire culture period and during $18 \mathrm{~h}$ of in vitro maturation (IVM) did not affect oocyte maturation progression, second meiotic spindle morphology and steroidogenesis of the cultured follicles (data not shown).

\section{Stimulation for ovulation and maturation}

IVM medium consisted of control media supplemented with $1.5 \mathrm{IU} / \mathrm{ml}$ recombinant hCG (rhCG) and $5 \mathrm{ng} / \mathrm{ml}$ recombinant human epidermal growth factor (rEGF) (Roche Diagnostics, Brussels, Belgium). Stimulus for maturation was performed as follows.

\section{Follicle-enclosed oocytes (FEO)}

On day 12 of culture in vitro grown follicles were stimulated with IVM media for $18 \mathrm{~h}$, serving as an ovulatory and maturation stimulus. 


\section{Isolated CEOs}

On day 12 of culture CEOs were isolated from their in vitro grown preovulatory follicles. CEOs were washed three times and placed in microdroplets of IVM media under oil for $18 \mathrm{~h}$.

\section{In vivo controls}

CEOs were obtained from antral follicles of 26-day-old mice $48 \mathrm{~h}$ after priming with $5 \mathrm{IU} / \mathrm{ml}$ pregnant mare serum gonadotrophin (Folligon; Intervet, Mechelen, Belgium). CEOs were placed in microdroplets of IVM media under oil for $18 \mathrm{~h}$.

\section{Assessment of hormonal production in vitro}

During follicle culture, $10 \mu \mathrm{l}$ samples from each culture droplet were collected on every other day from surviving follicles and were pooled per plate. Samples were stored at $-20^{\circ} \mathrm{C}$ for hormone analysis. Oestradiol- $17 \beta$ concentrations were measured using a commercially available direct radioimmunoassay from Clinical Assays (Oestradiol 2; Sorin Fueter, Brussels, Belgium). Progesterone concentrations were measured by direct radioimmunoassay (Progesterone Coatria) from bioMérieux (Marcy-l'Etoile, France). These immunoassays had been validated for this use previously (Cortvrindt et al. 1998).

\section{Processing oocytes for immunofluorescence analysis}

Oocytes were fixed and processed for microtubule detection and/or chromatin analysis as previously described (Wickramasinghe et al. 1991). Microtubules were labelled using a mixture of monoclonal anti- $\alpha$-tubulin (T-9026; Sigma) and anti- $\beta$-tubulin (T-4026; Sigma) at a final dilution of $1: 100$ for $60 \mathrm{~min}$ at $37^{\circ} \mathrm{C}$. The fluoresceinated secondary antibody, Alexa fluor-conjugated goat-antimouse IgG, was used at a 1:200 final dilution (A-11001; Molecular Probes, Eugene, OR, USA) for $60 \mathrm{~min}$ at $37^{\circ} \mathrm{C}$. Chromatin was visualized with ethidium homodimer-2 at a 1:2000 final dilution for $15 \mathrm{~min}$ (Molecular Probes). Processed oocytes were mounted in 90\% glycerolphosphate-buffered saline containing $0.2 \%$ DABCO (Diamino-bi-cyclo-octane) and analyzed by conventional fluorescence microscopy or laser scanning microscopy (Olympus; Omnilabo N.V., Aartselaar, Belgium, a gift from Serono International, Geneva, Switzerland).

\section{Experimental set-up}

Experiment 1: effect of PDE3-I on follicle development and on oocyte meiotic progression

In order to analyze the effects of long-term exposure to PDE3-I, follicle developmental rate and survival were recorded during culture in control media, in the presence of $1 \mu \mathrm{M}$ org9935 or $1 \mu \mathrm{M}$ cilostamide. Thereafter, oocytes from the cultured follicles were analyzed for the following. (a) Reversibility of PDE3 inhibition. On day 12 of follicle culture, oocytes were removed from their preovulatory follicles, mechanically denuded and placed in control media. After $18 \mathrm{~h}$, oocyte maturation (GV, GVBD; germinal vesicle breakdown, PB; polar body) and survival were assessed. PB extruded oocytes were fixed for spindle analysis. (b) Kinetics of meiotic progression. Alternatively, following 12-days of follicle culture, mechanically denuded oocytes were placed in control media and the kinetics of nuclear progression were evaluated during culture at $0,1,2,3,6,7,8,9,10$ and $18 \mathrm{~h}$.

\section{Experiment 2: oocyte spindle quality after reversal of PDE3 inhibition}

In order to evaluate oocyte quality and meiotic process following reversal from PDE3 inhibition, CEOs from 12-day follicle culture in either control media or in $1 \mu \mathrm{M}$ or $10 \mu \mathrm{M}$ org9935-supplemented media were isolated and underwent IVM for $18 \mathrm{~h}$. Simultaneously, intact follicles (FEO) cultured in control media were stimulated on day 12 as controls. Oocyte maturity and diameter were recorded and $\mathrm{PB}$ extruded oocytes were fixed for spindle analysis.

\section{Experiment 3: effect of PDE3 inhibition on steroid production and ovulation of FEO}

Follicles were cultured for 12 days in control media and in the presence of $1 \mu \mathrm{M}$ or $10 \mu \mathrm{M}$ org9935. Steroid production was analyzed during follicle development and after the ovulatory stimulus. At $18 \mathrm{~h}$ of IVM, the number of CEOs presenting mucification was recorded. The oocytes were denuded and maturation stages were assessed.

\section{Statistical analysis}

Differences in the proportion of follicles at each developmental stage and in the proportion of follicles that survived were calculated by Chi-square test (contingency analysis). Differences in oocyte diameter, in the proportion of mucified CEOs and in the proportion of oocytes at each of the meiotic stages were examined using one-way ANOVA. Percentages were statistically analyzed after arcsine transformation. Differences in concentrations of steroid production during culture, over time and in response to treatment dose were assessed using two-way ANOVA. When ANOVA indicated a significant difference $(P<0.05)$, the Tukey post-hoc test was performed to determine differences between treatment means. Variation among replicates is expressed as the S.E.M.

\section{Results}

\section{Experiment 1: effect of PDE3-I on follicle development and on oocyte meiotic progression}

Possible adverse effects of PDE3-Is on follicle development and survival were tested. The growth progression 

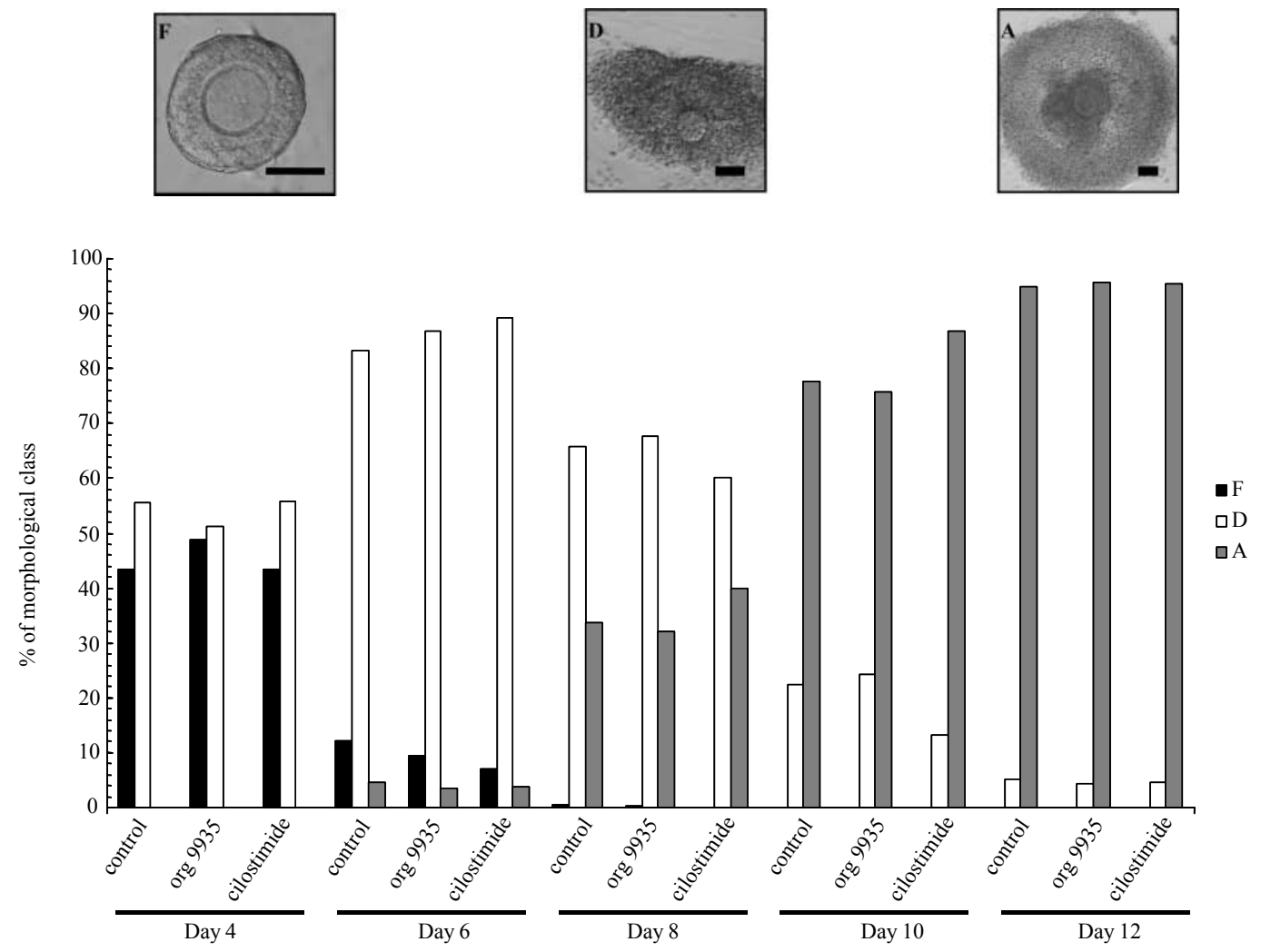

Figure 1 Morphogenesis of early preantral follicles. (F) Follicle enclosed within intact basement membrane, (D) follicle diffused with granulosa cells growing out of the basement membrane and (A) antral-like follicular stage with a centrally located oocyte/cumulus surrounded by a less dense area and a more dense rim of mural granulosa cells. Percentages were calculated over the surviving follicles cultured in control media $(n=175)$ or $1 \mu \mathrm{M}$ org9935- $(n=284)$ or $1 \mu \mathrm{M}$ cilostamide-supplemented media $(n=131)$ from four independent replicates (Chi-squared analysis, $P>0.05)$. Scale bars $=50 \mu \mathrm{m}$.

was not altered by the presence of the PDE3-Is org9935 or cilostamide (Fig. 1) ( $\mathrm{P}>0.05)$. On day 4 of culture, theca and granulosa cells began to proliferate, and by day 6 more than $80.0 \%$ of the follicle population for each culture condition had a diffused appearance, with layers of granulosa and theca cells sustaining the oocyte. The proportion of follicles with an antral-like cavity, which started to appear on day 6 but became prominent from day 8 onwards, was similar for all the cultures (Fig. 1). Follicle survival, which is characterized by oocytes retained within the follicular structure without signs of granulosa cells and oocyte degeneration, was revealed to be similar among the treatment conditions ( $\sim 95 \%)$.

\section{Reversibility of PDE3 inhibition}

The addition of org9935 or cilostamide during the 12-day follicle culture did not affect the potential of the oocytes for spontaneous reinitiation of meiosis $18 \mathrm{~h}$ after inhibitor withdrawal and IVM (Fig. 2). Only a few oocytes did not progress to PB extrusion $(17.7 \pm 8.0$ and $15.5 \pm 5.1 \%$ of follicles cultured with $1 \mu \mathrm{M}$ org9935 and $1 \mu \mathrm{M}$ cilostamide), which was similar to the control $(21.2 \pm 8.6 \%)$ $(P>0.05)$. As shown in Table $1,70.7 \%$ of in vitro grown oocytes in controls that extruded a PB $18 \mathrm{~h}$ after IVM had an MII spindle versus 74.3 and $87.5 \%$ of oocytes cultured in org9935 and in cilostamide $(P>0.05)$. The highest proportion of PB oocytes displaying an MII configuration $(96.9 \%)$ was from the in vivo control group $(P<0.05)$.



Figure 2 Meiotic status of denuded oocytes following 12-day follicle culture and $18 \mathrm{~h}$ of IVM. Oocytes were denuded after isolation from follicles cultured for 12 days with or without PDE3-I. Data are the mean \pm S.E.M. \% of the total number of oocytes following $18 \mathrm{~h}$ of IVM (one-way ANOVA, P>0.05). deg; degenerated. 
Table 1 Incidence of metaphase II (MII) plate in denuded oocytes $18 \mathrm{~h}$ after IVM.

\begin{tabular}{lc}
\hline Conditions & MII/PB oocytes $(\%)$ \\
\hline In vitro control* & $46 / 65(70.7)^{\mathrm{a}}$ \\
$1 \mu \mathrm{M}$ org 9935* & $52 / 70(74.3)^{\mathrm{a}}$ \\
$1 \mu \mathrm{M}$ cilostamide* & $21 / 24(87.5)^{\mathrm{ab}}$ \\
In vivo control & $62 / 64(96.9)^{\mathrm{b}}$ \\
\hline
\end{tabular}

a,b Values with different superscripts denote significant differences (Chi-squared analysis, $P<0.05$ ).

*Oocytes isolated from 12-day follicle culture.

\section{Kinetics of meiosis progression}

The kinetics of meiotic resumption is shown in Fig. 3. The time-span for resumption of meiosis and $\mathrm{PB}$ extrusion in oocytes following a 12-day follicle culture and $18 \mathrm{~h}$ of IVM differed slightly among the experimental groups. The oocytes started to reinitiate meiosis in culture after $2 \mathrm{~h}$. Although the time for meiosis resumption (GVBD) seemed longer for oocytes grown in vitro in $1 \mu \mathrm{M}$ org9935supplemented medium, the percentages of GVBD reached similar values in the following hours of culture compared with the other groups. At $7 \mathrm{~h}$ of culture, $30.7 \%$ of GVBD oocytes grown in the presence of cilostamide extruded the first $\mathrm{PB}$, while only a few oocytes from the in vitro control and org9935 cultures extruded a PB. After $10 \mathrm{~h}$ in culture, the proportion of PB extruded oocytes in the cilostamide group reached similar values compared with the other groups. Finally, at $18 \mathrm{~h}$ of culture, the proportion of PB extruded oocytes did not differ between the oocytes cultured in the different conditions (Fig. 3).

\section{Experiment 2: oocyte spindle quality after reversal of PDE3 inhibition}

Since in the previous experiment both PDE3-Is resulted in similar findings, we opted to focus further experiments on varying doses of only org9935. In this experiment, oocytes were isolated as CEOs, thus approaching a more physiological condition for IVM. After removal from 12-day PDE3-I cultures, isolated CEOs were able to resume meiosis within a time-frame of $18 \mathrm{~h}$ of IVM (Fig. 4). At this time, the proportion of oocytes arrested at the GV stage was similarly low in all the groups, thus an increased concentration of org9935 $(10 \mu \mathrm{M})$ was not harmful for oocyte meiosis reinitiation. No differences in the ability to resume meiosis were found among oocytes derived from the different conditions. PB extrusion was $\sim 80.0 \%$ in all groups (Fig. 4 ).

Oocyte diameter was measured to determine if the presence of different concentrations of org9935 could influence oocyte growth. The final diameter of PB extruded oocytes did not differ between oocytes from the control conditions (FEO-stimulated, $72.6 \pm 0.8 \mu \mathrm{M}$ and CEOstimulated, $72.3 \pm 0.8 \mu \mathrm{M}$ ) and org9935 cultures (CEOstimulated $1 \mu \mathrm{M}, 72.1 \pm 0.8 \mu \mathrm{M}$ and CEO-stimulated $10 \mu \mathrm{M}, 72.5 \pm 0.7)$.

Microtubules and chromosome configuration were analyzed to assess whether the CEOs reached the MII stage with a well-formed spindle in a normal time-frame. As indicated in Table 2, a large proportion of oocytes attained the MII stage with a morphologically normal spindle after 12-day follicle culture and $18 \mathrm{~h}$ of IVM (Fig. 5) and few oocytes in all the conditions were at the pre-MIl stages (e.g. telophase I). Spindles of a normal morphology included the symmetric spindles forming a slight barrelshaped network of microtubules presenting broad spindle poles or the symmetric spindles comprising a barrelshaped form and more narrowed spindle poles. These morphological characteristics could be observed in a similar proportion in the MII-stage oocytes in all the conditions. The increased concentration of inhibitor to $10 \mu \mathrm{M}$ throughout all the culture period was not deleterious to oocytes at this investigated end-point. Moreover, in vitro growth of oocytes for 12 days in the presence of the

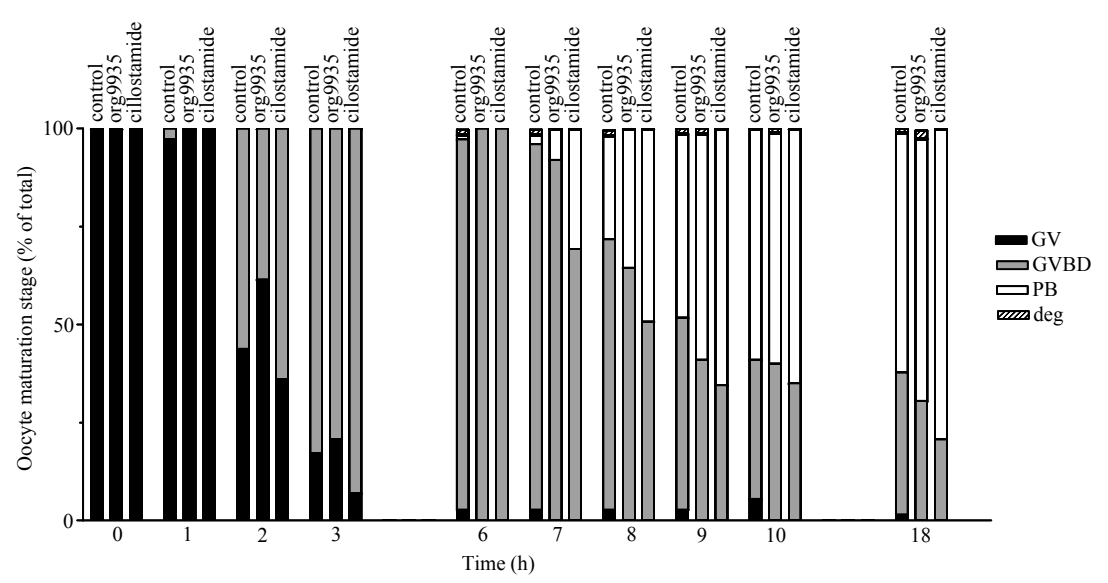

Figure 3 Effect of 12-day culture with PDE3-I on the kinetics of meiosis progression of denuded oocytes during IVM. Oocytes were isolated from follicles cultured for 12 days in control medium $(n=75)$ or $1 \mu \mathrm{M}$ org9935- $(n=73)$ or $1 \mu \mathrm{M}$ cilostamide-supplemented media $(n=72)$. Data are presented as the mean $\%$ of the total number of oocytes. Error bars are omitted for the sake of clarity (one-way ANOVA, $P>0.05$ ). deg; degenerated. 


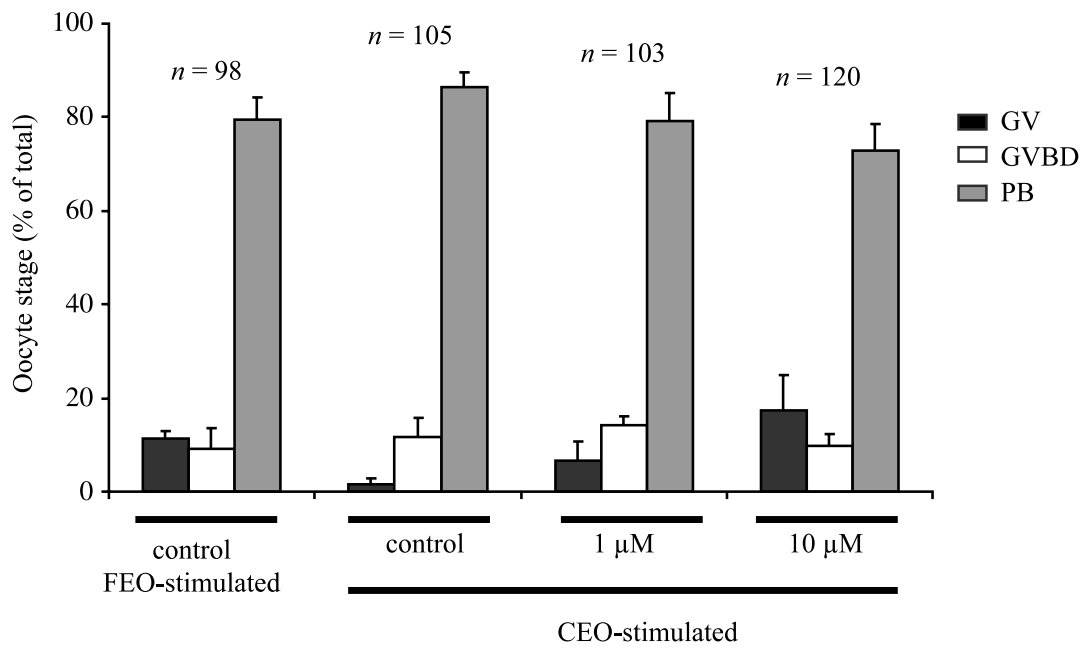

Figure 4 Effect of 12-day follicle culture with PDE3-I on the maturation of CEOs after $18 \mathrm{~h}$ of IVM. CEO-stimulated: CEOs were removed from follicles that had been cultured in control media or $1 \mu \mathrm{M}$ or $10 \mu \mathrm{M}$ org9935-supplemented media, and which underwent IVM with rhCG/rEGF. FEO-stimulated: intact follicles cultured in control media for 12 days were stimulated with rhCG/rEGF for $18 \mathrm{~h}$. Data are the mean \pm S.E.M. $\%$ of the total number of oocytes of three independent replicates (oneway ANOVA, $P>0.05)$.

higher org9935 concentration did not affect the chromosome arrangement and, in all the groups, a high proportion of oocytes had well-aligned chromosomes at the equator of the spindle (Fig. 5C). Only a few oocytes from all conditions presented spindles with abnormal chromosome alignment, in which one or more chromosomes were scattered along the equator.

\section{Experiment 3: effect of PDE3 inhibition on steroid production and in vitro ovulation of FEOs}

In order to investigate whether PDE3 inhibition influences steroidogenesis of granulosa cells by any non-specific action in the granulosa cells or, indirectly, via its action in the oocyte, oestrogen and progesterone production were quantified during follicle and oocyte growth. Although the mean concentration of oestradiol was higher when the PDE3-I, org9935, was used at $10 \mu \mathrm{M}$ compared with the other groups, it did not reach a level of significance (Fig. 6A). For both PDE3-I doses tested, oestradiol levels increased gradually throughout the culture period and became significantly increased from day 10 onwards over day 6 levels. The mean oestradiol-17 $\beta$ concentrations were similar in all the conditions for each day of culture.

The presence of PDE3-I did not influence the production of progesterone throughout the culture period (Fig. 6B). The rhCG/rEGF administration on day 12 caused a significant increase in progesterone concentrations on day 13 for all culture conditions (Fig. 6B).

The rate of development throughout the 12 days of culture was unchanged by the addition of PDE3-I and at least $94.0 \%$ of antral-like follicles survived at the end of culture $(P>0.05)$. After the ovulatory stimulus, a large majority of CEOs (about 95.0\%) in all the conditions was very expanded showing that the presence of PDE3-I during the entire culture and during $18 \mathrm{~h}$ of IVM by rhCG/rEGF did not influence the capacity for mucification in vitro. Mucified CEOs were usually detached from their differentiated mural-like granulosa cells (Fig. 7). Although, at this time, even with their cumulus cells expanded, $98.2 \pm 1.2$ and $97.0 \pm 1.8 \%$ of oocytes from 12-day cultures with $1 \mu \mathrm{M}$ and $10 \mu \mathrm{M}$ org9935 remained blocked at the GV stage compared with controls $(P<0.001)$ (Fig. 8). Those GV-arrested oocytes were left to mature in order to check their viability, and after a further $20 \mathrm{~h}$ of incubation in control medium the oocytes were capable of resuming meiosis up to PB extrusion in $86.6 \pm 1.7$ and $85.1 \pm 4.0 \%$ from $1 \mu \mathrm{M}$ and $10 \mu \mathrm{M}$ org9935 cultures respectively.

\section{Discussion}

In the ovarian follicle, the action of PDE isoforms induces intra-follicular cAMP hydrolysis contributing to differences in local distribution of this nucleotide within the cellular

Table 2 Assessment of meiotic progression, spindle normality and chromosome alignment in oocytes after 12-day follicle culture and $18 \mathrm{~h}$ of IVM. ${ }^{\text {a } V a l u e s ~ a r e ~ m e a n ~} \pm$ S.E.M. \% of three independent replicates.

\begin{tabular}{lccr}
\hline In vitro conditions & $\begin{array}{c}\text { PB extruded } \\
\text { oocytes analyzed }\end{array}$ & $\begin{array}{c}\text { Telophase I } \\
\text { stages }^{\mathrm{a}}\end{array}$ & $\begin{array}{c}\text { Normal MII } \\
\text { spindle }^{\mathrm{a}}\end{array}$ \\
\hline FEO-stimulated control & 53 & $6.4 \pm 6.5$ & $87.8 \pm 4.5$ \\
CEO-stimulated control* & 75 & $7.3 \pm 4.5$ & $88.5 \pm 4.1$ \\
CEO-stimulated org9935 $(1 \mu \mathrm{M})^{*}$ & 69 & $7.8 \pm 8.5$ & $86.4 \pm 2.1$ \\
CEO-stimulated org9935 $(10 \mu \mathrm{M})^{*}$ & 60 & $7.9 \pm 6.6$ & $86.1 \pm 5.5$ \\
\hline
\end{tabular}

a No significant differences were observed among the conditions (one-way ANOVA, $P>0.05$ ).

* CEO were isolated from their antral-like cavity follicles on day 12 and placed in IVM media. 

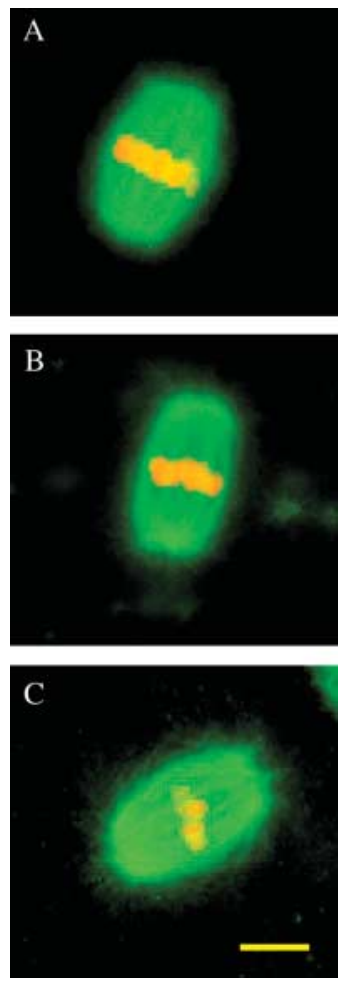

Figure 5 Representative fluorescent images of spindles of mouse oocytes grown in vitro. CEOs underwent IVM for $18 \mathrm{~h}$ following removal from 12-day follicle culture in $(\mathrm{A})$ control media, in the presence of (B) $1 \mu \mathrm{M}$ or (C) $10 \mu \mathrm{M}$ org9935. Bar $=10 \mu \mathrm{M}$.

compartments (Thomas et al. 2002, Park et al. 2003). To date, several reports attest the efficiency of selective PDEIs to arrest oocyte meiosis when applied in a short culture period during follicle and oocyte development (Tsafriri et al. 1996, Mayes \& Sirard 2002, Thomas et al. 2002, Jensen et al. 2002, Nogueira et al. 2003b). The present results have revealed that long-term exposure of germ cells and their somatic follicular cells to the PDE3 specific inhibitors, cilostamide or org9935, does not alter their normal development. In line with the specificity of their action, the PDE3-Is displayed no direct effects on the somatic cells. Gonadotrophin-dependent proliferation and formation of an antral-like cavity was unaffected, as shown by the identical pattern of follicle differentiation among the groups. After removal from PDE3-I-containing medium, reinitiation of meiosis occurred without obvious changes in oocyte morphology.

In mice, in vivo grown oocytes acquire meiotic competence in the late preantral follicle stage (Sorensen \& Wassarman 1976). Under the present culture conditions, the oocytes, which are physiologically arrested at prophase I, acquired competence to resume meiosis from day 8 of culture onward (transition between late preantral and antral formation) (Cortvrindt et al. 1998). The application of two sorts of PDE3-I during the entire culture did not impair functions related to the subsequent activation of MPF, following inhibitor removal, since oocytes were able to progress to MII. However, our results showed that a difference exists between oocytes grown in vitro and in vivo in a higher proportion of MII among in vivo matured oocytes, which suggests that the in vitro microenvironment provided for oocyte growth and maturation remains suboptimal to support the correct development of oocyte competency (Eppig et al. 1994, Cortvrindt \& Smitz 2002).

Several studies corroborate that successful fertilization and embryo development depend not only on the nuclear maturity of the oocyte but also on the cytoplasmic maturation. Oocyte 'quality' can be approached in several ways including spindle structure and chromosome segregation. Spindle integrity in oocytes is an important prerequisite for the formation of a cytogenetically balanced embryo (Eichenlaub-Ritter 2000).

The meiotic spindle concentrates components responsible for guiding a balanced distribution of chromosomes during asymmetric cytokinesis, which will be reflected later in the embryo. Therefore, it was judged important to detect whether applying the inhibitor during these prolonged periods would cause changes in spindle structure and chromosome alignment. Chromosomes were well aligned at the equator of the MII spindle, demonstrating that fidelity of chromosome segregation was successfully achieved in all groups, even when a higher dose of org9935 $(10 \mu \mathrm{M})$ was used. In this study, the prolonged use of the inhibitor did not adversely affect spindle structure. While we did not strictly compare the morphometric differences among the groups, most spindles in PDE3-Itreated oocytes and in vitro controls were similar in having barrel-shaped microtubular structures.

The time-course for resumption of meiosis upon mechanical removal of cumulus cells revealed no differences among the in vitro controls and the inhibitor-treated groups. The meiotic cell cycle is regulated by a series of phosphorylations and dephosphorylations co-ordinating check-point proteins at each stage of meiotic division. Genes and proteins are stored during oocyte growth and sequentially translated and activated during specific steps of meiotic maturation. It was not within the objective of our study to investigate the time-course of each phase during the progression of meiosis I (GVBD, MI, anaphase and telophase I) and, therefore, further time-course experiments could more precisely determine whether at any stage of meiosis the processes are changed.

Bi-directional communication between the germ cell and its surrounding steroidogenic cells is crucial not only for oocyte development but also for normal granulosa cell function (Vanderhyden et al. 1993, Eppig et al. 1997). FSH-induced gene transcription in granulosa cells is, at least partially, mediated by cAMP via the PKA pathway. PKA phosphorylates the nuclear protein cAMP-response element binding protein, which binds to the cAMPresponse element, stimulating transcription of, for instance, the genes for P450arom (CYP19) and mediates transcription of the $\mathrm{LH}$ receptor (Chen et al. 2000). By 


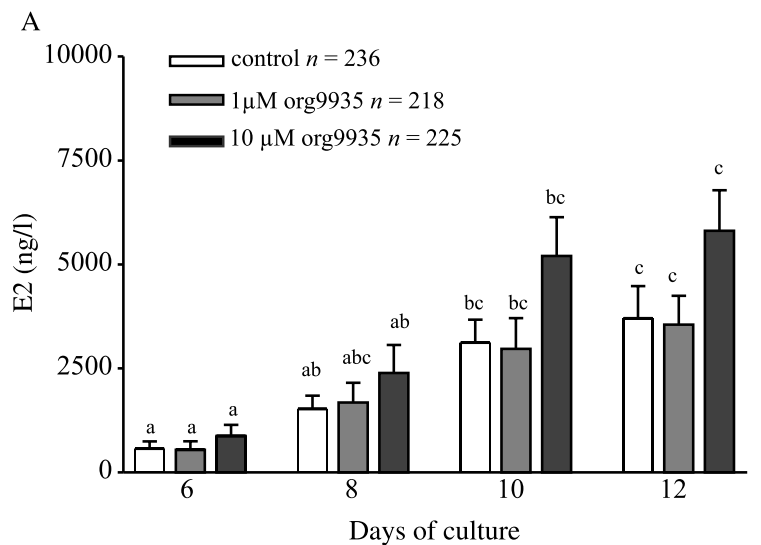

B

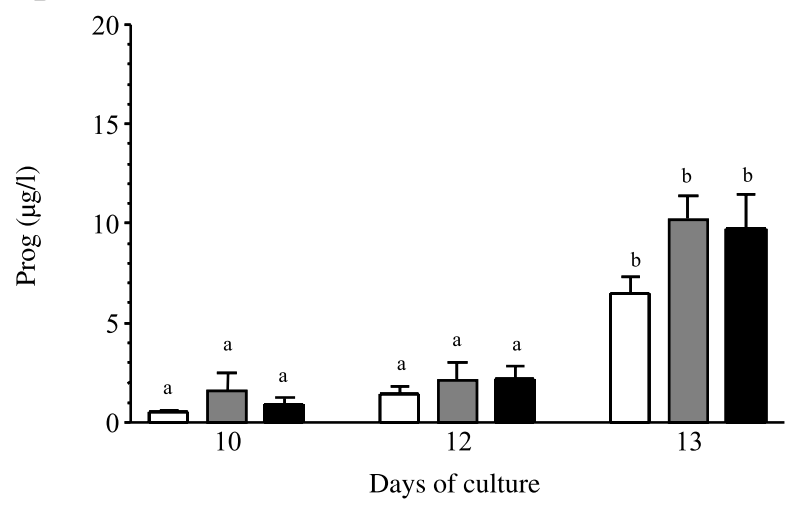

Figure 6 Analysis of conditioned medium collected from follicles during growth. (A) Oestradiol-17 $\beta$ (E2) secreted up to 12 days of follicle culture. (B) Basal progesterone (Prog) concentrations on days 10 and 12 of culture and after rhCG/rEGF administration on day 13. Results are the mean \pm S.E.M. concentrations of steroids of four independent replicates. No differences were observed between the individual treatments for each day of culture (days 6, 8, 10 or 12 of culture). Means within the same treatment with no common superscripts are significantly different over culture time (two-way ANOVA, $P<0.05$ ).

virtue of the expression of P450arom and 17ß-hydroxysteroid dehydrogenase, granulosa cells become highly active in converting theca-derived androgens to oestradiol. The first step in the biosynthetic pathway to progesterone, namely conversion of cholesterol to pregnenolone by P450scc is normally dependent on the cAMP pathway during follicle development. The results have shown that, in the presence of a PDE3 specific inhibitor, granulosa cell differentiation pattern in response to gonadotrophin occurs normally. Although the mean oestradiol concentration tended to be higher when $10 \mu \mathrm{M}$ PDE3-I was used, there were no significant differences in oestradiol nor in progesterone secretion patterns of inhibitor-treated follicles versus controls. This suggested that aromatase and P450scc gene induction via the cAMP signalling pathway was not altered. Recently, Park et al. (2003) reported that PDE4D-deficient mice have defective ovulation and alterations in CAMP accumulation in response to gonadotrophin, with significantly lower and long-lasting levels of CAMP produced in response to hCG compared with
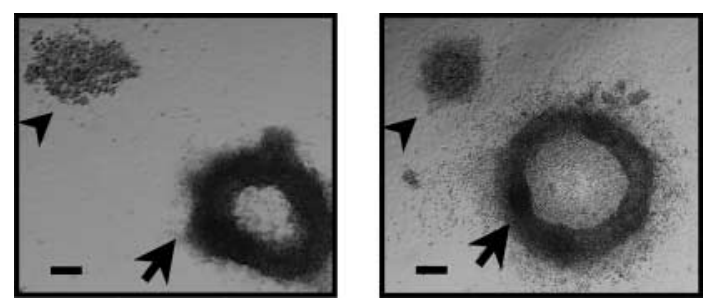

Figure 7 In vitro mucification of CEO (arrowheads) upon stimulation by rhCG/rEGF following 12-day follicle culture in control media (left) or in org9935-supplemented media (right) (bar $=50 \mu \mathrm{m})$. Arrows indicate mural cells forming the antral-like cavity.

wild-type mice. The present data add to the evidence that the inhibition of PDE3 in follicles has no impact on the functioning of the somatic cell component and that the presumed elevation of cAMP into the oocyte has no influence on steroid biosynthesis. In agreement with our results, Thomas et al. (2002) demonstrated that PDE3-I applied in combination with rFSH failed to induce an increase in cAMP levels in mural granulosa cells. In contrast, rolipram, a specific PDE4 inhibitor used in combination with $\mathrm{rFSH}$ caused an augmentation in cAMP levels in mural granulosa cells.

Mammalian oocytes secrete regulatory factors to the development and function of the surrounding granulosa cells in the ovarian follicles (Eppig et al. 2002). Data generated from in vitro studies have shown that paracrine signals secreted by the oocyte induce cumulus expansion (e.g. mucification) via cumulus cell MAPK-dependent mechanisms in response to FSH and EGF (Buccione et al. 1990, Salustri et al. 1990, Su et al. 2002) and suppression of mRNA LH receptor expression in granulosa cells (Eppig et al. 1997). The present results have indicated that by maintaining continuous exposure to PDE3-I, mechanisms related to cumulus expansion and steroidogenic

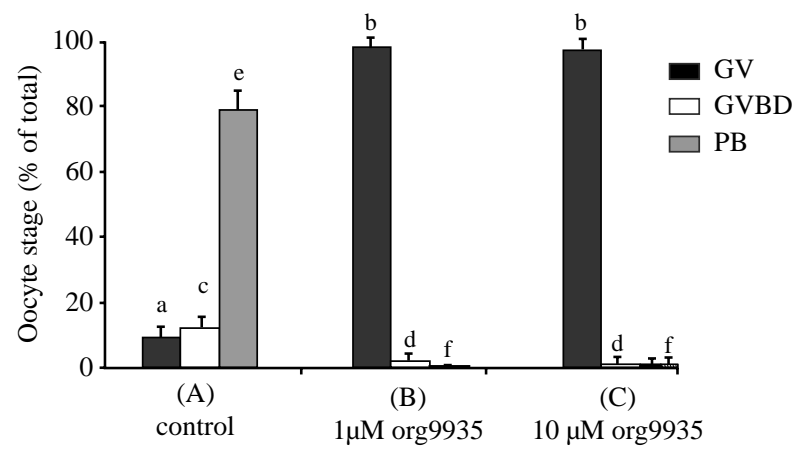

Figure 8 Effect of PDE3-I on meiosis progression of stimulated FEOs. Stimulation for in vitro ovulation was administered on FEOs cultured for 12 days in (A) controls, (B) $1 \mu \mathrm{M}$ or (C) $10 \mu \mathrm{M}$ org9935-supplemented media. Oocytes were denuded to evaluate maturation $18 \mathrm{~h}$ after stimulus with rhCG/rEGF. Data are presented as the mean \pm S.E.M. $\%$ of the total number of oocytes of three independent replicates. Means within the same meiotic stage with no common superscripts are significantly different $\left(\mathrm{GV}^{\mathrm{a}-\mathrm{b}}, \mathrm{GVBD}^{\mathrm{c}-\mathrm{d}}, \mathrm{PB}^{\mathrm{e}-\mathrm{f}}\right.$; oneway ANOVA, $P<0.05$ ). 
oocyte-secreted factors also remained functionally unaltered upon rhCG/rEGF stimulation on day 12 of culture.

In rodents, EGF can reverse the meiotic arrest effect by hypoxanthine on CEO, an effect mediated by cumulus cells (Downs et al. 1988), and can induce cumulus expansion in CEOs. When specific PDE3-I cilostamide is used, EGF is unable to induce maturation in isolated rodents CEOs (Coticchio et al. 2004). Previous work has shown that the addition of rEGF in FEOs in inhibitor-free cultures optimizes hCG-induced maturation (Smitz et al. 1998). It is now known that, within the ovulatory follicle, LH/hCG indirectly cause cumulus expansion and oocyte maturation by inducing expression of EGF-like growth factors in mural granulosa cells and these factors overcome the inhibitory effect of hypoxanthine on meiosis (Park et al. 2004). Presently, by applying rhCG/rEGF on the preovulatory follicle, the inhibitory effect of the PDE3-I could not be overcome, at least not within a period of $18 \mathrm{~h}$ after administration, which is the normal time-frame to complete meiosis in mouse. Although mucification of cumulus and meiotic resumption mechanisms closely parallel each other in vivo, these processes can be stimulated independently from each other. This result also confirmed the recently reported data that oocytes of PDE3-deficient mice are ovulated at the GV stage (Masciarelli et al. 2004).

In conclusion, we have shown that the continuous prolonged in vitro exposure of FEOs to PDE3 specific inhibitors does not alter somatic cell functionality, differentiation or oocyte development. Providing withdrawal from the prolonged PDE3-I culture, oocytes are highly capable of resuming meiosis and form a morphologically normal MII spindle. Further study is warranted to investigate whether a long-term exposure of oocytes to PDE3-I is also innocuous to fertilization and embryonic development. The results suggested that PDE3-I can be selected when systems comprising an extension of the oocyte culture period to improve ooplasmic maturation are required.

\section{Acknowledgements}

This work was supported by funds from Novo Nordisk A/S, Denmark. The authors acknowledge the help of Johan Schiettecatte and Anne Gerard from the laboratory of radioimmunology at the Academic Hospital of the Vrije Universiteit Brussel for the hormone analysis. The authors are grateful to Dr Claire Bourgain and Dr David Keefe for a critical reading of the manuscript. The authors declare that there is no conflict of interest that would prejudice the impartiality of this scientific work.

\section{References}

Allworth AE \& Albertini DF 1993 Meiotic maturation in cultured bovine oocytes is accompanied by remodeling of the cumulus cell cytoskeleton. Developmental Biology 158 101-112.

Bilodeau-Goeseels S 2003 Effects of phosphodiesterase inhibitors on spontaneous nuclear maturation and cAMP concentrations in bovine oocytes. Theriogenology 60 1679-1690.
Buccione R, Vanderhyden BC, Caron PJ \& Eppig JJ 1990 FSHinduced expansion of the mouse cumulus oophorus in-vitro is dependent upon a specific factor(s) secreted by the oocyte. Developmental Biology 138 16-25.

Canipari R 2000 Oocyte-granulosa cell interactions. Human Reproduction Update 6 279-289.

Chen S, Liu X \& Segaloff DL 2000 A novel cyclic adenosine 3', $5^{\prime}-$ monophosphate-responsive element involved in the transcriptional regulation of the lutropin receptor gene in granulosa cells. Molecular Endocrinology 14 1498-1508.

Cortvrindt RG \& Smitz JE 2002 Follicle culture in reproductive toxicology: a tool for in-vitro testing of ovarian function? Human Reprouction Update 8 243-254.

Cortvrindt RG, Hu Y, Liu J \& Smitz JE 1998 Timed analysis of the nuclear maturation of oocytes in early preantral mouse follicle culture supplemented with recombinant gonadotropin. Fertility and Sterility 70 1114-1125.

Coticchio G, Rossi G, Borini A, Grondahl C, Macchiarelli G, Flamigni C, Fleming S \& Cecconi S 2004 Mouse oocyte meiotic resumption and polar body extrusion in vitro are differentially influenced by FSH, epidermal growth factor and meiosis-activating sterol. Human Reproduction 19 2913-2918.

Dekel N 1996 Protein phosphorylation/dephosphorylation in the meiotic cell cycle of mammalian oocytes. Reviews of Reproduction $182-88$.

Downs SM, Daniel SA \& Eppig J 1988 Induction of maturation in cumulus cell-enclosed mouse oocytes by follicle-stimulating hormone and epidermal growth factor: evidence for a positive stimulus of somatic cell origin. Journal of Experimental Zoology 245 86-96.

Eichenlaub-Ritter U 2000 The determinants of nondisjunction and their possible relationship to oocyte ageing. In Female Reproductive Ageing, vol 1, pp 149-184. Eds TE Velde, PL Pearson, FG Broekmans. New York: Parthenon Publishing.

Elvin JA, Yan C, Wang P, Nishimori K \& Matzuk MM 1999 Molecular characteristics of the follicle defects in the growth differentiation factor 9-deficient ovary. Molecular Endocrinology 13 1018-1034.

Eppig JJ, Schultz RM, O'Brien M \& Chesnel F 1994 Relationship between the developmental programs controlling nuclear and cytoplasmic maturation of mouse oocytes. Developmental Biology $1641-9$.

Eppig JJ, Wigglesworth K, Pendola F \& Hirao Y 1997 Murine oocytes suppress expression of luteinizing hormone receptor messenger ribonucleic acid by granulosa cells. Biology of Reproduction 56 976-984.

Eppig JJ, Wigglesworth K \& Pendola F 2002 The mammalian oocyte orchestrates the rate of ovarian follicular development. PNAS 99 2890-2894.

Jensen JT, Schwinof KM, Zelinski-Wooten MB, Conti M, DePaolo LV \& Stouffer RL 2002 Phosphodiesterase 3 inhibitors selectively block the spontaneous resumption of meiosis by macaque oocytes in-vitro. Human Reproduction 17 2079-2084.

Joyce IM, Pendola FL, O'Brien M \& Eppig JJ 2001 Regulation of prostaglandin-endoperoxide synthase 2 messenger ribonucleic acid expression in mouse granulosa cells during ovulation. Endocrinology 142 3187-3197.

Masciarelli S, Horner K, Liu C, Park SH, Hinckley M, Hockman S, Nedachi T, Jin C, Conti M \& Manganiello V 2004 Cyclic nucleotide phosphodiesterase $3 \mathrm{~A}$-deficient mice as a model of female infertility. Journal of Clinical Investigation 14 196-205.

Mayes MA \& Sirard MA 2002 Effect of type 3 and type 4 phosphodiesterase inhibitors on the maintenance of bovine oocytes in meiotic arrest. Biology of Reproduction 66 180-184.

Nogueira D, Albano C, Adriaenssens T, Cortvrindt R, Bourgain C, Devroey P \& Smitz J 2003a Human oocytes reversibly arrested in prophase I by phosphodiesterase type 3 inhibitor in-vitro. Biology of Reproduction 69 1042-1052.

Nogueira D, Cortvrindt R, De Matos DG, Vanhoutte L \& Smitz J $2003 b$ Effect of phosphodiesterase type 3 inhibitor on 
developmental competence of immature mouse oocytes in-vitro. Biology of Reproduction 69 2045-2052.

Park JY, Richard F, Chun SY, Park JH, Law E, Horner K, Jin SL \& Conti M 2003 Phosphodiesterase regulation is critical for the differentiation and pattern of gene expression in granulosa cells of the ovarian follicle. Molecular Endocrinology 17 1117-1130.

Park JY, Su YQ, Ariga M, Law E, Jin SL \& Conti M 2004 EGF-like growth factors as mediators of $\mathrm{LH}$ action in the ovulatory follicle. Science 303 682-684.

Richard FJ, Tsafriri A \& Conti M 2001 Role of phosphodiesterase type $3 \mathrm{~A}$ in rat oocyte maturation. Biology of Reproduction $\mathbf{6 5}$ $1444-1451$.

Salustri A, Yanagishita M \& Hascall VC 1990 Mouse oocytes regulate hyaluronic acid synthesis and mucification by FSH-stimulated cumulus cells. Developmental Biology 138 26-32.

Shitsukawa K, Andersen CB, Richard FJ, Horner AK, Wiersma A, van Duin M \& Conti M 2001 Cloning and characterization of the cyclic guanosine monophosphate-inhibited phosphodiesterase PDE3A expressed in mouse oocyte. Biology of Reproduction 65 $188-196$

Smitz J, Cortvrindt R \& Hu Y 1998 Epidermal growth factor combined with recombinant human chorionic gonadotrophin improves meiotic progression in mouse follicle-enclosed oocyte culture. Human Reproduction 13 664-669.

Sorensen RA \& Wassarman PM 1976 Relationship between growth and meiotic maturation of the mouse oocyte. Developmental Biology $50531-536$.

Su YQ, Wigglesworth K, Pendola FL, O'Brien MJ \& Eppig JJ 2002 Mitogen-activated protein kinase activity in cumulus cells is essential for gonadotropin-induced oocyte meiotic resumption and cumulus expansion in the mouse. Endocrinology 143 2221-2232.
Thomas RE, Armstrong DT \& Gilchrist RB 2002 Differential effects of specific phosphodiesterase isoenzyme inhibitors on bovine oocyte meiotic maturation. Developmental Biology 244 215-225.

Thomas RE, Thompson JG, Armstrong DT \& Gilchrist RB 2004 Effect of specific phosphodiesterase isoenzyme inhibitors during in-vitro maturation of bovine oocytes on meiotic and developmental capacity. Biology of Reproduction 71 1142-1149.

Tsafriri A, Chun SY, Zhang R, Hsueh AJ \& Conti M 1996 Oocyte maturation involves compartmentalization and opposing changes of cAMP levels in follicular somatic and germ cells: studies using selective phosphodiesterase inhibitors. Developmental Biology $178393-402$.

Vanderhyden BC, Cohen JN \& Morley P 1993 Mouse oocytes regulate granulosa cell steroidogenesis. Endocrinology 133 423-426.

Wickramasinghe D, Ebert KM \& Albertini DF 1991. Meiotic competence acquisition is associated with the appearance of M-phase characteristics in growing mouse oocytes. Developmental Biology 143162 -172. Erratum in Developmental Biology 1991144220.

Wiersma A, Hirsch B, Tsafriri A, Hanssen RG, Van de Kant $M$, Kloosterboer HJ, Conti M \& Hsueh AJ 1998 Phosphodiesterase 3 inhibitors suppress oocyte maturation and consequent pregnancy without affecting ovulation and cyclicity in rodents. Journal of Clincal Investigation $102532-537$.

Received 16 January 2004

First decision 1 March 2005

Revised manuscript received 21 April 2005

Accepted 20 May 2005 